\title{
2D Simulations of Deflagrations in White Dwarfs
}

\author{
By ELI LIVNE
}

Steward Observatory, University of Arizona, Tucson, AZ 85721, USA

\section{Introduction}

The convective deflagration mechanism in white dwarfs became widely used to explain Type Ia Supernovae (SNIa) due the success of a single model, namely model W7 of Nomoto et al.(1984). Problems concerning the deflagration model were discussed by Sutherland \& Wheeler (1984), Nomoto et al.(1984), Woosley \& Weaver (1986), Woosley (1990), Khokhlov (1991a) and others. The main question however is still: does the deflagration model have any hydrodynamical basis, or, could the assumed Rayleigh Taylor instability accelerate the flame to the required speed?

Nomoto et al.(1984) estimated the turbulent speed from the mixing length arguments to be

$$
v_{t} \approx(g l / 2)^{\frac{1}{2}}
$$

where $v_{t}$ is the turbulent speed, $g=G M \delta \rho /\left(r^{2} \rho\right)$ is the effective acceleration, and $l$ is the length of the largest unstable modes, usually of the order of the pressure scale height. The relevance of mixing length theory to the deflagration problem is at least questionable. Two different effects may enhance the burning rate, relative to the laminar speed, when the front is unstable. First, mixing of burnt and unburnt matter can widen the front and accelerate its propagation like other dissipation mechanisms (this requires that perturbations, of the size of the front width, be unstable), and secondly, as the front becomes wrinkled, the total burning rate increases in proportion to the surface (Woosley 1990). We now discuss these two effects.

\section{Linear Stability of the Deflagration Front}

The analysis of the stability of ablation fronts, formed in shells accelerated by laser beams, show that the growth rate can be approximated (Takabe et al.1985) by :

$$
\omega(k)=\alpha(k g)^{1 / 2}-\beta k v_{f},
$$

where $\alpha=0.9, \beta=3$ to 4 and $v_{f}$ is the front speed. Due to the presence of an extra linear term there is a critical wave number, $k_{c}=\alpha^{2} g /\left(\beta v_{f}\right)^{2}$, above which all modes are stable. Moreover, the growth rates of all other modes are lower than that of the Rayleigh Taylor case, which has a maximum at $k_{\max }=0.25 k_{c}$.

Deflagration fronts have the same structure as ablation fronts, as the only difference being the different source of energy. For the early stages of the burning in a WD, $g \approx 2 \cdot 10^{9} \mathrm{~cm} \mathrm{~s}^{-2}$ and $v_{f} \approx 100 \mathrm{~km} \mathrm{~s}^{-1}$, so that perturbations with $l<5 \mathrm{~km}$ are stable while larger scales are unstable. Since the width of the front is less than $1 \mathrm{~cm}$ early on, this implies that locally the front propagates with the laminar speed. The most unstable perturbations have scales of $20 \mathrm{~km}$ at the early stages of the burning, and their growth rate is about half the Rayleigh Taylor growth rate. The conductive (laminar) flame speed 
is approximated (Timmes \& Woosley 1992) by:

$$
v_{c}=92.0\left(\frac{\rho}{2 \cdot 10^{9}}\right)^{0.805} \mathrm{~km} \mathrm{~s}^{-1}
$$

for an initial composition of $X\left({ }^{12} C\right)=0.5$.

\section{2D Numerical Simulations}

We performed many 2D simulations of the burning front using several methods. These simulations extend our study into the nonlinear regime and test the importance of the surface effect. The flame propagates in these simulations according $v_{f}=\max \left(v_{c}, v_{t}\right)$, where the turbulent speed $v_{t}$ is given by

$$
v_{t}=\left[\left(\nabla \Phi \cdot D^{\perp}\right) \frac{\delta \rho}{2 \rho}\right]^{\frac{1}{2}} .
$$

Here $\Phi$ is the gravitational potential and $D^{\perp}$ is a vector having the length of the cell face and direction normal to the face.

Coarse grid results were presented in Livne (1993), where we used 90 zones in the radial direction and 41 zones in the angular direction on one quadrant. Subsequent calculations, which used $180 \times 90$ and $260 \times 140$ zones in the radial and angular direction, respectively, showed similar results. Four snapshots of temperature contours, obtained from the high resolution sequence, are shown in figure 1. The typical pattern of these calculations shows a slow growth of the initial perturbation together with merging of smaller structures to bigger ones. A faster mushroom shape structure along the axis of symmetry is an artifact of the imposed axial symmetry. After roughly one second, expansion becomes dominant and the total burning rate drops slowly. At two seconds there is no more significant burning while the star is still bound. Simulations in which the turbulent flow is set to zero yield less than $0.3 \cdot 10^{51} \mathrm{erg}$, which is roughly twice as much as the energy produced in the spherical case, and is still much less than the binding energy of the WD $\left(0.51 \cdot 10^{51} \mathrm{erg}\right)$. When the turbulent speed is included in the simulation, the unperturbed case yields $0.3 \cdot 10^{51}$ erg while the perturbed cases produce 2 times more, which was hardly enough to unbind the star but not to generate anything like a SNIa, as it leaves an unacceptable amount of unburnt C-O.

\section{Implications on explosion mechanisms}

The fate of the explosion is determined by the competition between expansion, which suppresses the burning rates, and the increase of the total burning rate due to the increase of the surface of the front. Our calculations always show that the instability is too slow to enhance the burning rate to the value needed by an acceptable model for SNIa.

The results support the "delayed detonation" mechanism, suggested by Khokhlov (1991a,b) and Woosley (1991). As the deflagration front dies, or at least slows down considerably, Rayleigh Taylor instability is recovered and mixing occurs on all scales. This mixing can be enhanced if the star is bound at this stage, and the positive velocity slope becomes negative. We suggest that this small scales mixing is the trigger for the transition to detonation (after Khokhlov 1991b). The discussion above implies that the transition occurs not by accelerating the front but by slowing it down. Since this occurs at relatively low densities, the condition for obtaining good agreement with observations is automatically fulfilled.

The impact of the detonation wave on the large nonspherical structures formed in 

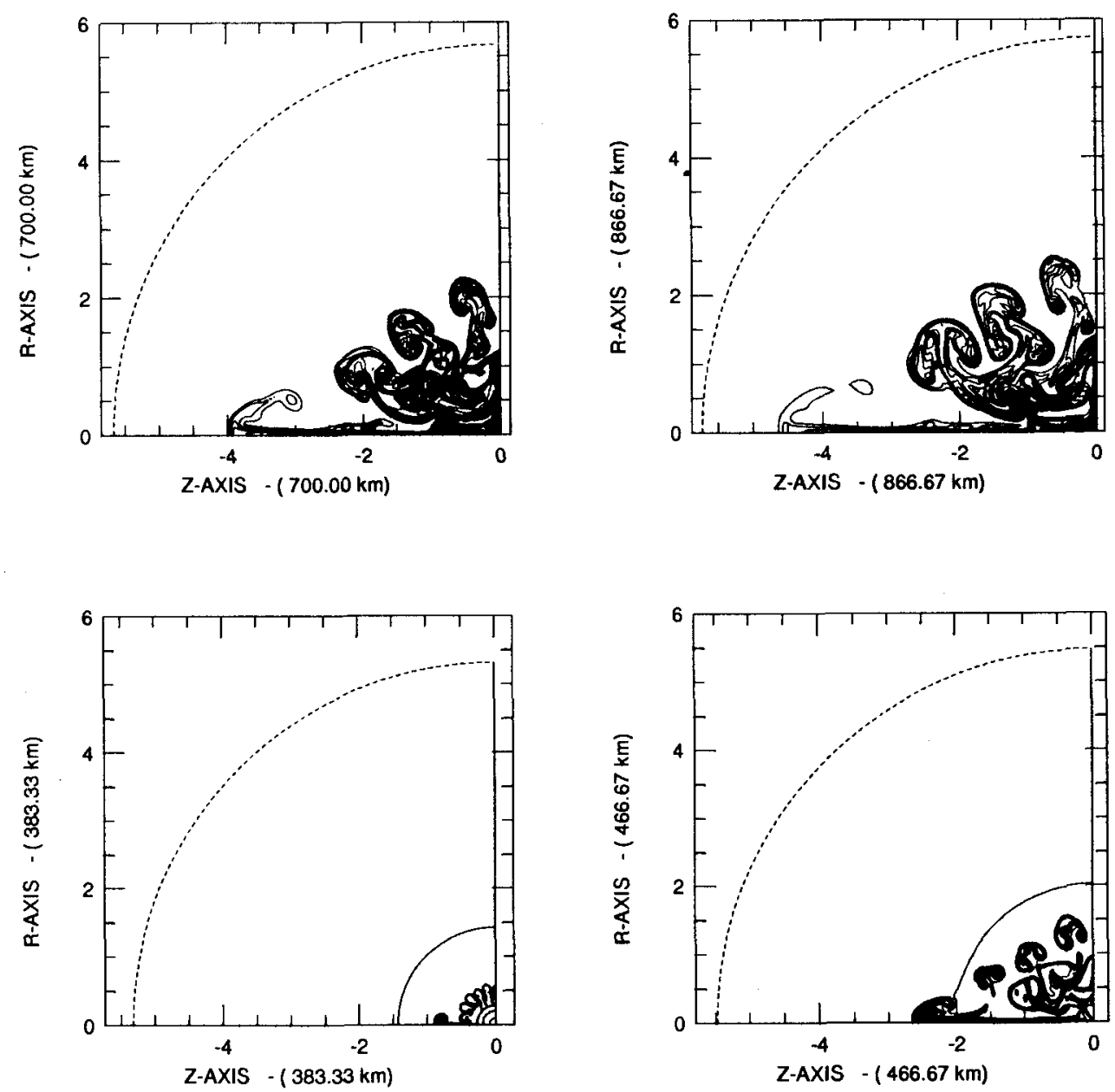

Figure 1. Temperature contours at $\mathrm{t}=0.5 \mathrm{sec}$ (left bottom), $\mathrm{t}=1 \mathrm{sec}$ (right bottom), $\mathrm{t}=1.5$ $\sec$ (left top) and $t=1.9 \mathrm{sec}$ (right top). The dashed line represents the surface of the WD.

the first stage of the burning may lead to a significant mixing and may bring elements of the iron group to the outer layers. More work is needed in order to better understand the transition to detonation. For further implications of the $2 \mathrm{D}$ motions upon the nucleosynthesis and the amount of neutronization see a paper by D. Arnett in this volume.

\section{Acknowledgements}

I wish to thank Dave Arnett, Alexei Khokhlov, Stan Woosley and Adam Burrows for may useful discussions.

\section{REFERENCES}

Khokhlov, A. M. 1991a, A\&A, 245, 114.

Khokhlov, A. M. 1991b, A\&A, 245, L25.

Livne, E. 1993, ApJL, 406, L20. 
Nomoto, K., Thielemann, F. K. \& Yokoi, K. 1984, ApJ, 286, 644.

Sutherland, P. G, \& Wheeler, J. C. 1984, ApJ, 280, 282.

Takabe, H., Mima, K., Montierth, L., \& Morse, R. L. 1985, Phys. Fluids, 28, 3676.

Timmes, F. X. and Woosley, S. E. 1992, ApJ, 396, 649.

Woosley, S. E. 1991, in 'Gamma-Ray Line Astrophysics', ed P. Durouchoux \& N. Prantzos, New York: American Institute of Physics, 270.

Woosley, S. E. \& Weaver., T. A. 1986, in 'Radiation Hydrodynamics in Stars and Compact Objects', Springer Verlag, D. Mihalas and K. H. Winkler eds. 